\title{
How to produce accurate inelastic cross sections from an indirect measurement method?
}

\author{
Maëlle Kerveno $^{1, *}$, Greg Henning ${ }^{1}$, Catalin Borcea ${ }^{2}$, Philippe Dessagne ${ }^{1}$, Marc Dupuis ${ }^{3}$, Stéphane Hilaire ${ }^{3}$, \\ Alexandru Negret ${ }^{2}$, Markus Nyman ${ }^{4}$, Adina Olacel $^{2}$, Eliot Party ${ }^{1}$, and Arjan Plompen ${ }^{4}$ \\ ${ }^{1}$ Université de Strasbourg CNRS, IPHC UMR 7178, Strasbourg, France \\ ${ }^{2}$ Horia Hulubei National Institute of Physics and Nuclear Engineering, Bucharest-Magurele, Romania \\ ${ }^{3}$ CEA, DAM, DIF, Arpajon, France \\ ${ }^{4}$ European Commission, Joint Research Centre, Geel, Belgium
}

Received: 31 October 2017 / Received in final form: 8 March 2018 / Accepted: 14 May 2018

\begin{abstract}
Inelastic reactions $((\mathrm{n}, \mathrm{xn})$ for $x \geq 1)$ play a key role in reactor cores as they influence the slowing down of the neutrons. A reactor neutron energy spectrum depends thus on this process which is in strong competition with elastic scattering and fission; a nice example is the case of ${ }^{238} \mathrm{U}$. Inelastic scattering $(x=1)$ impacts $k_{\text {eff }}$ and radial power distribution in the nuclear reactor. For several years, it has been shown that the knowledge of the inelastic cross sections in nuclear databases is not good enough to accurately simulate reactor cores and a strong demand for new measurements has emerged with very tight objectives (only a few percent) for the uncertainties on the cross section. To bypass the well-known experimental difficulty to detect neutrons, the prompt $\gamma$-ray spectroscopy method is a powerful but indirect way to obtain inelastic cross sections. Our collaboration has used this method for more than ten years and have produced a lot of $\left(n, n^{\prime} \gamma\right)$ cross sections for nuclei from ${ }^{7} \mathrm{Li}$ to ${ }^{238} \mathrm{U}$. In this article, we will first discuss the issues of the prompt $\gamma$-ray spectroscopy regarding the control of all the uncertainties involved in the $\left(n, n^{\prime} \gamma\right)$ cross section estimation. Secondly, we will focus on the role of theoretical modeling which, in certain cases, is crucial to reach the objectives of a few percent uncertainty on the $\left(\mathrm{n}, \mathrm{n}^{\prime}\right)$ cross sections.
\end{abstract}

\section{Introduction}

For new generation reactor development or optimization of fuel cycles and operating procedures, quality of nuclear data is the basic prerequisite for accurate simulations. Among processes affecting neutron population and energy distribution in a reactor, neutron inelastic scattering $\left(\mathrm{n}, \mathrm{n}^{\prime}\right)$ - is particularly important as it acts as a slowing down process. Further, the $(\mathrm{n}, \mathrm{xn})$ reactions result in neutron multiplication. Moreover, neutron inelastic scattering is a key reaction which influences the radial power distribution and $k_{\text {eff }}$ core parameters. Nevertheless, it has been shown that the knowledge of these cross sections is not satisfactory to ensure accurate core parameters calculations as seen in references [1-3]. This situation leads finally to several requests in the High Priority Request List of NEA/OECD [4]. For example, the demand concerning the ${ }^{238} \mathrm{U}$ inelastic scattering is to reduce by a factor of two [3] or four [1] the uncertainty which reaches $20 \%$ in current evaluated nuclear data files.

\footnotetext{
* e-mail: maelle.kerveno@iphc.cnrs.fr
}

The collaboration of three teams from CNRS (France), EC-JRC-Geel (Belgium) and IFIN-HH (Romania) has developed, fifteen years ago, two experimental setups dedicated to precise measurements of neutron inelastic scattering cross sections at the neutron time-of-flight facility GELINA operated by EC-JRC-Geel [5]. Setups are based on the prompt $\gamma$-ray spectroscopy method coupled to time-of-flight measurements. This method allows the measurements of $\gamma$-production cross sections $-(\mathrm{n}, \mathrm{xn} \gamma)-$ which are used in a second step to determine the total (n,xn) cross section using level and decay sequence information from literature. This method is thus considered as an indirect method, unlike direct methods that detect secondary neutrons, and the deduced total $(\mathrm{n}, \mathrm{xn})$ cross section is often a lower limit for energies above the energy of the highest level that can be observed by $\gamma$-deexcitation. Nevertheless, this technique can provide a large set of $(\mathrm{n}, \mathrm{xn} \gamma)$ cross sections which constitute severe tests and constraints for theoretical models. The question is therefore, from the various partial cross section measurements, how can we accurately determine the total cross section which fully satisfy the level of accuracy requested by the applications? In the following section, we will detail 
the experimental method. The third section is devoted to the description of the procedure that we have elaborated to maximize the control of uncertainties and minimize their magnitudes. In the fourth section, a discussion is proposed about the tools we develop to produce accurate $(\mathrm{n}, \mathrm{xn})$ reaction cross sections from the measured $(\mathrm{n}, \mathrm{xn} \gamma)$ cross section. And finally, conclusions and perspectives end the paper.

\section{Indirect experimental method}

\subsection{The $(n, x n \gamma)$ technique}

The $(\mathrm{n}, \mathrm{xn})$ reactions can be studied by three experimental methods based either on the detection of emitted neutrons or $\gamma$ 's. Each method, direct or indirect, has its own advantages and disadvantages [5] in terms of detection difficulties, corrections to apply, neutron beams suitability, etc. Our collaboration has chosen the prompt $\gamma$-ray spectroscopy method which is an indirect one, but which allows to perform experiments at white neutron sources using time-of-flight measurements. With this technique, the $\gamma$-rays coming from the de-excitation of the nucleus formed by the $(\mathrm{n}, \mathrm{xn})$ process, are detected and the $(\mathrm{n}, \mathrm{xn} \gamma)$ cross sections can be deduced. The detection time of the $\gamma$-rays is used to deduce, thanks to the pulsation of the accelerator, the in-beam neutron time-of-flight, which is related to the incident neutron energy. From these measured cross sections, two ways are possible to produce the $(\mathrm{n}, \mathrm{xn})$ cross sections as shown schematically in Figure 1.

The first possibility is to use the structure information (level scheme of the nucleus of interest, branching ratios and internal conversion coefficients if necessary) to deduce the experimental $(\mathrm{n}, \mathrm{xn})$ cross sections from the measured cross sections. Indeed, the total $(\mathrm{n}, \mathrm{xn})$ cross section is equal to the sum of all the partial cross sections of $\gamma$-transitions that feed the ground state (GS). If a $\gamma$-transition to GS from an excited level is not detected for various experimental reasons but another one from the same level is observed, then the transition to GS can be deduced using the branching ratio (if known) and used for the determination of the total $(\mathrm{n}, \mathrm{xn})$ cross section. Nevertheless, in practice, not all the $(n, x n \gamma)$ cross sections for transitions to GS can be measured over the entire neutron energy range, thus the obtained total cross sections is often a lower limit. More precisely, for $x>1$, the GS can be produced directly (no $\gamma$-ray emission) and the method provides thus always a lower limit of the total cross section. For $x \geq 1$, due to physics, measurement issues and limited knowledge of the decay of the nucleus, one can miss some transitions to the GS.

To bypass this limitation, another way is to use nuclear reaction codes (Fig. 1). The principle is to constrain and tune the "free" nuclear model parameters with the measured $(\mathrm{n}, \mathrm{xn} \gamma)$ cross sections and then produce a validated total $(\mathrm{n}, \mathrm{xn})$ cross section with the nuclear reaction code.

For both methods and in the context of accuracy issues, some key questions arise. First of all, experimentalists have to produce accurate $(\mathrm{n}, \mathrm{xn} \gamma)$ cross sections meaning that all the uncertainty sources have to be identified, controlled

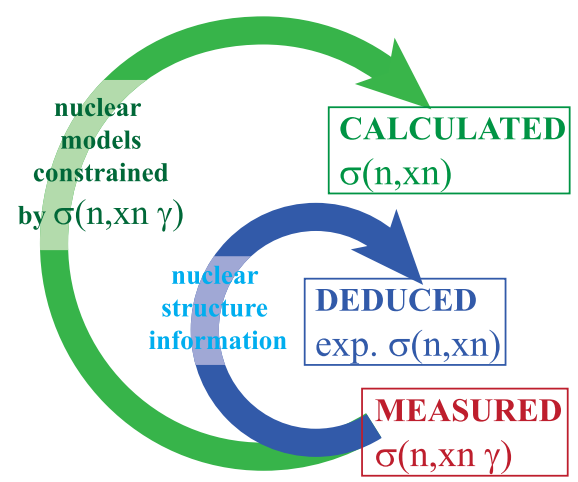

Fig. 1. Schematic view of the two ways to deduce total $(\mathrm{n}, \mathrm{xn})$ cross section from measured $(\mathrm{n}, \mathrm{xn} \gamma)$ ones.

and minimized. Covariance and correlation information have also to be produced to give relevant data to theoreticians and evaluators. The good knowledge of nuclear structure can be questioned too, since this is an essential component of the two manners for $(\mathrm{n}, \mathrm{xn})$ cross section calculation. Finally, in the second method, as the parameters of nuclear models are tuned to the experimental $(\mathrm{n}, \mathrm{xn} \gamma)$ cross sections, the theoretical calculated $(\mathrm{n}, \mathrm{xn})$ cross section could be produced with uncertainties but it is rarely the case.

The pillar of this method is thus the measurement of the $(\mathrm{n}, \mathrm{xn} \gamma)$ cross section with a good accuracy. A short description of the experimental setup and the analysis procedure is then given in the following section before the focus on the uncertainties management in the next section.

\subsection{Experimental setup for $(n, x n \gamma)$ measurement}

There are two HPGe setups for $(\mathrm{n}, \mathrm{xn} \gamma)$ measurements at GELINA, called GRAPhEME and GAINS, respectively used for measurements on actinides and on stable nuclei. More details can be found in references [5-7] and references therein. In this section, we use as an example of GRAPhEME setup (GeRmanium array for Actinides PrEcise MEasurements), which is installed at neutron flight path 16 of the GELINA facility, $30 \mathrm{~m}$ distance from the neutron source. It is composed of six HPGe planar detectors placed at $110^{\circ}$ and $150^{\circ}$ with respect to the neutron beam direction, which are nodes of the fourth Legendre polynomial. This configuration allows the use of the Gaussian quadrature to perform exact integration of the $\gamma$-ray angular distribution for $\gamma$-transitions with multipolarity up to three. Due to the very good energy resolution of the germanium detectors, selective identification of the detected $\gamma$-ray allows the reduction of the ambiguity to the underlying nuclear process. The incoming neutron flux is measured using a ${ }^{235} \mathrm{U}$ ionization chamber and time-of-flight measurements allow the determination of the incident neutron energy distribution. The partial cross section at angle $\theta$ can be thus addressed, for each $\gamma$-transition of interest, with the following formula:

$$
\frac{d \sigma}{d \Omega}\left(\theta_{i}, E_{n}\right)=\frac{1}{4 \pi} \frac{N_{G E}\left(\theta_{i}, E_{n}\right)}{N_{F C}\left(E_{n}\right)} \frac{\varepsilon_{F C}}{\varepsilon_{G E}} \frac{\varsigma_{F C}}{\varsigma_{\text {sple }}} \sigma_{F}\left(E_{n}\right),
$$


where $N_{G E}$ and $N_{F C}$ represent the dead time corrected numbers of counts, respectively, for a given $\gamma$-ray in the HPGe energy spectrum and for the fission chamber counts above the discrimination threshold. $\varepsilon_{G E}$ and $\varepsilon_{F C}$ are the Germanium detector's and the fission chamber's efficiencies. $\sigma_{F}$ is the ${ }^{235} \mathrm{U}$ fission cross section and $\varsigma_{F C}$ and $\varsigma_{\text {sple }}$ are the areal densities (atoms $/ \mathrm{cm}^{2}$ ) of ${ }^{235} \mathrm{U}$ in the fission chamber and nuclei of interest in the sample. Gamma energy distributions are produced for time-of-flight gates. The widths of the time gates are adjusted to cover several time bins (10 ns) to improve statistics (at the expense of neutron energy resolution). The neutron energy resolution, at $30 \mathrm{~m}$ from the neutron source, is $0.17 \%$ at $30 \mathrm{keV}$, worsen as energy increases and reaches almost $5 \%$ at high neutron energy. To produce as accurate as possible $(\mathrm{n}, \mathrm{xn} \gamma)$ cross sections, the first step is to precisely quantified and minimize the uncertainties of all parameters involved in equation (1). The following section describes the work performed on this subject and the results obtained for the uncertainty of each ingredient of the cross section formula.

\section{Accurate determination of $(n, x n y)$ cross sections}

\subsection{Experimental uncertainties}

To reach the requested statistical target uncertainty, long measurement periods are usually necessary. This is due to the generally low magnitude cross sections, the low detection efficiency of HPGe detectors, and the limited size of the samples used. It is especially the case for actinides, for which the $\gamma$-energy distribution is often complex with a mix of $\gamma$-rays from background (radioactivity) or de-excitation of fission products. Very good statistics is mandatory to perform efficient peak identification and integration. Typically, measurements take from a few hundred up to a couple of thousand hours of beam time. These long measurements can be sensitive to the stability of the data acquisition system and therefore a careful runs screening is necessary to obtain, for each detector, optimal raw data sets.

In neutron-induced experiments, the absolute normalization of the data is a key point of the analysis and it implies a significant uncertainty contribution due to the use of a secondary reaction for the neutron flux determination. In our case, we have chosen the fission reaction on ${ }^{235} \mathrm{U}$, which is an IAEA standard and is wellknown in the $0.1-20 \mathrm{MeV}$ neutron energy range. The uncertainty on the ${ }^{235} \mathrm{U}(\mathrm{n}, \mathrm{f})$ cross section ranges from $0.6 \%$ to $1.4 \%$ between 0.1 and $20 \mathrm{MeV}$. The ionization chamber efficiency has been well characterized by the combination of measurements, simulation and calibration as explained in reference [6]. Finally, the neutron flux can be determined with an average uncertainty around $2.5 \%$.

For the HPGe $\gamma$-efficiency determination, we have developed a procedure based on source measurements and MCNP simulations [8]. The neutron beam impinging on the sample has a diameter is $5.5 \mathrm{~cm}$. Thus two ${ }^{152} \mathrm{Eu}$ sources, a point one and an extended one, are used for calibration runs. These runs then serve to adjust the geometry (size of

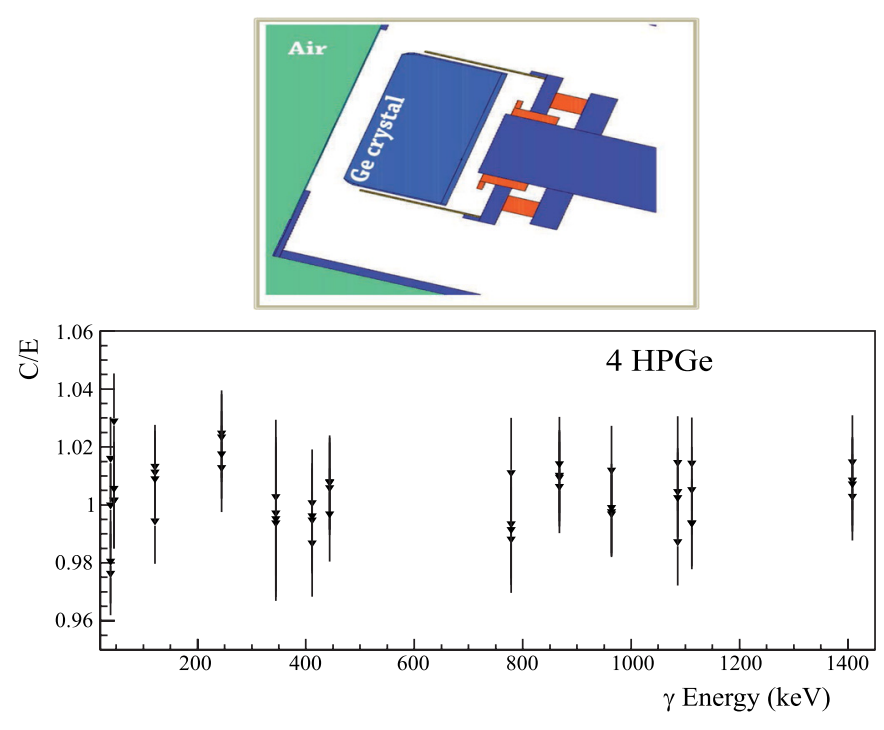

Fig. 2. On the top is represented the adjusted geometry of a HPGe planar crystal obtained during the $\mathrm{C} / \mathrm{E}$ optimization (bottom) procedure for $\gamma$-efficiency calibration.

the active part, shape of the dead layers or position) of the Ge crystal in a MCNPX-2.6 simulation. This adjustment is performed very carefully to obtain a simulated over experimental efficiency ratio $(\mathrm{C} / \mathrm{E}$ ratio) roughly constant over the whole $\gamma$-energy range. This ratio obtained for four planar detectors of GRAPhEME is shown in Figure 2 together with the resulting geometry of the simulated crystal. The dispersion of the $\mathrm{C} / \mathrm{E}$ ratio is then used to assess the $\gamma$-efficiency uncertainty resulting in a typical value of $2 \%$. One can remark that this uncertainty is dominated by the uncertainty coming from the initial activity of the ${ }^{152} \mathrm{Eu}$ sources which is $1.4 \%$.

Once the $\gamma$-efficiency is well characterized, the last parameter to determine is the number of atoms in the sample. To do this, we use as prior estimation, the information provided by the EC-JRC-Geel target preparation laboratory that weighted and measured the size of the sample. As a typical example, for a ${ }^{n a t} \mathrm{U}$, the mass, the diameter and the thickness are given respectively with a relative uncertainty of $10^{-3} \%, 4.10^{-2} \%$ and $3.3 \%$. The use of active samples allows to determine directly the number of atoms of interest in the sample. Indeed we determine the number of atoms from the number of counts in $\gamma$-peaks from radioactivity (corrected by the $\gamma$-efficiency) registered during off-beam runs. This supplies a mass measurement for each $\gamma$-peak as shown in Figure 3 in agreement $(0.4 \%)$ with the weighted mass. Finally, the average number of ${ }^{238} \mathrm{U}$ nuclei is known with an uncertainty of $3 \%$.

All these uncertainties lead to a total uncertainty for the $(\mathrm{n}, \mathrm{xn} \gamma)$ cross sections ranging from $\sim 4 \%$ to $20 \%$ or more when the statistics is very low due to, for example, measurement time or available neutron flux. If one wants to decrease the final uncertainty, it is necessary to have more precisely calibrated sources and improve the method for the determination of the number of atoms in the sample. During the development of GRAPhEME, the analysis 


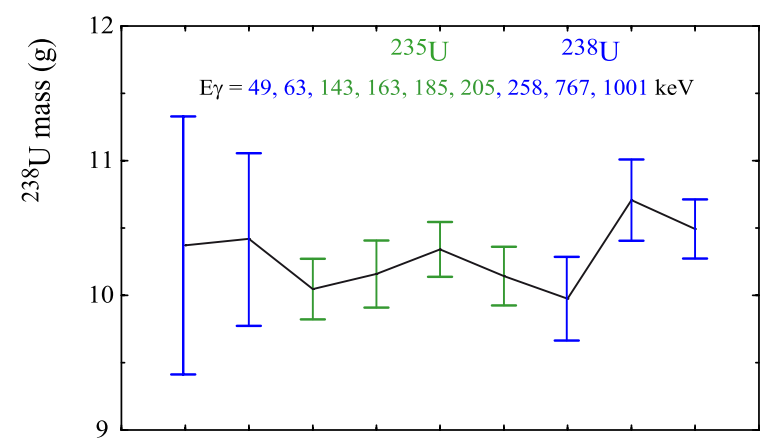

Fig. 3. Calculated ${ }^{238} \mathrm{U}$ mass as a function of the analysed $\gamma$-peaks from radioactivity of the sample.

procedure and the uncertainties treatment was originally performed with deterministic method. A new MC approach has been recently developed as described in the next section.

\subsection{Deterministic versus Monte Carlo method for uncertainty treatment}

To enrich the analysis procedure related to the GRAPhEME setup and to deeper understand and manage the uncertainty issues in our work, we have recently developed a new analysis program based on the Monte Carlo method for the $(n, x n \gamma)$ cross section determination. In this program, all the parameters involved in the $(\mathrm{n}, \mathrm{xn} \gamma)$ cross sections are varied randomly within probability distributions. For the fission chamber and $\gamma$-efficiencies, a Gaussian distribution is considered with a standard deviation of $2.1 \%$ and $2 \%$ respectively. The pile-up correction factor for fission chamber and HPGe detectors is also varied in a Gaussian distribution within a few percent. The number of atoms is varied in a Gaussian distribution with standard deviation of $2 \%$. The Monte Carlo analysis gives also the possibility to take into account the source of the uncertainty related to the time determination which is less direct in the deterministic ones. This effect is simulated with flat distributions by $\pm 5 \mathrm{~ns}$ for the fission chamber events and $\pm 10 \mathrm{~ns}$ (sampling period) for the HPGe events. The cross section is calculated for each set of parameters and a number of about 25 iterations is needed to reach convergence. The result for a $\gamma$-transition $(111 \mathrm{keV}$ in ${ }^{184} \mathrm{~W}$ ) is shown in Figure 4 . Due to the discrete time distribution of gamma events (in $10 \mathrm{~ns}$ wide bins), some combinations of parameters can lead to quantified increases in the calculated cross section, in particular on the edges, as one can see at high neutron energy in Figure 4.

Best uncertainty assessment requires the comparison between two standard analysis procedures that are the deterministic and the Monte Carlo. In our case, the two methods have been developped independantly by different person and then, we analyzed the uncertainty behavior and magnitudes in each method for the $122 \mathrm{keV} \gamma$-transition in ${ }^{186} \mathrm{~W}$. For the deterministic method, the cross section uncertainty is calculated taking into account classical error propagation through equation (1). The statistical uncertainty (related to the number of counts) and the systematic uncertainties (coming from detector efficiencies, number of atoms in the target and $(\mathrm{n}, \mathrm{f})$ cross section) are estimated separately. The statistical uncertainty takes into account the pile-up correction uncertainty which can reach $2 \%$. In the Monte Carlo method, the statistical uncertainty is related to the error on $\gamma$ and neutron counts. The systematic one is associated to the dispersion of the cross section results obtained after MC calculations. Figure 5 shows the relative uncertainties (total, statistical and systematic) obtained by the two methods. The overall behavior (high uncertainties at low and high neutron energy and plateau up to $5 \mathrm{MeV}$ ) of the total uncertainty is in agreement between the two methods. One can notice also that for the MC treatment, the statistical error magnitude is slightly lower than for the one estimated by deterministic approach where the pile-up uncertainty is included in the statistical uncertainty. A significant difference is observed between systematic errors which are almost constant in the deterministic method and dependent of the neutron energy in $\mathrm{MC}$ ones. This dependency can be mainly attributed to the dispersion on the time determination which is taken into account in the $\mathrm{MC}$ method and not in the deterministic one.

In conclusion, the two methods give similar results in magnitude but, as all uncertainties are not treated in the same way, some differences arise.

\subsection{Covariance and correlation matrices with Monte Carlo method}

In such experiment the source of correlations are numerous and arise from different effects as common parameters (the sample mass, the $\gamma$-efficiency which is determined with the same calibrated sources or the neutron flux) are using for each angular differential cross section determination. This contributes to strong correlation when the total $(\mathrm{n}, \mathrm{xn} \gamma)$ cross section is calculated via the Gauss quadrature. A first investigation of covariance and correlation determination has been performed for the GAINS setup in the case of ${ }^{56} \mathrm{Fe}$ $\left(n, n^{\prime} \gamma\right)$ reaction as explained in reference [9]. For the GRAPhEME setup, it was not yet done but the development of the new MC analysis program described in the previous section, gives us the opportunity to start this study. Indeed an asset of the Monte Carlo method is the possibility to produce almost directly the covariance and correlation matrices associated with the cross section determination. After Monte Carlo processing, each individually calculated cross section is stored and then processed with the NumPy python package [10] to produce covariance and correlation matrices. Figure 6 displays the correlation matrix for the $111 \mathrm{keV} \gamma$-transition cross section in ${ }^{184} \mathrm{~W}$ which is representative of posterior matrices. As expected, the cross sections are fully correlated, positively or negatively, between low and high neutron energies. This correlation is due to time determination uncertainty simulated by time variation (i.e. limits of the time windows) considering the number of observed $\gamma$ 's as obviously constant. More investigations and tests are planned for deeper understanding of these phenomena. 


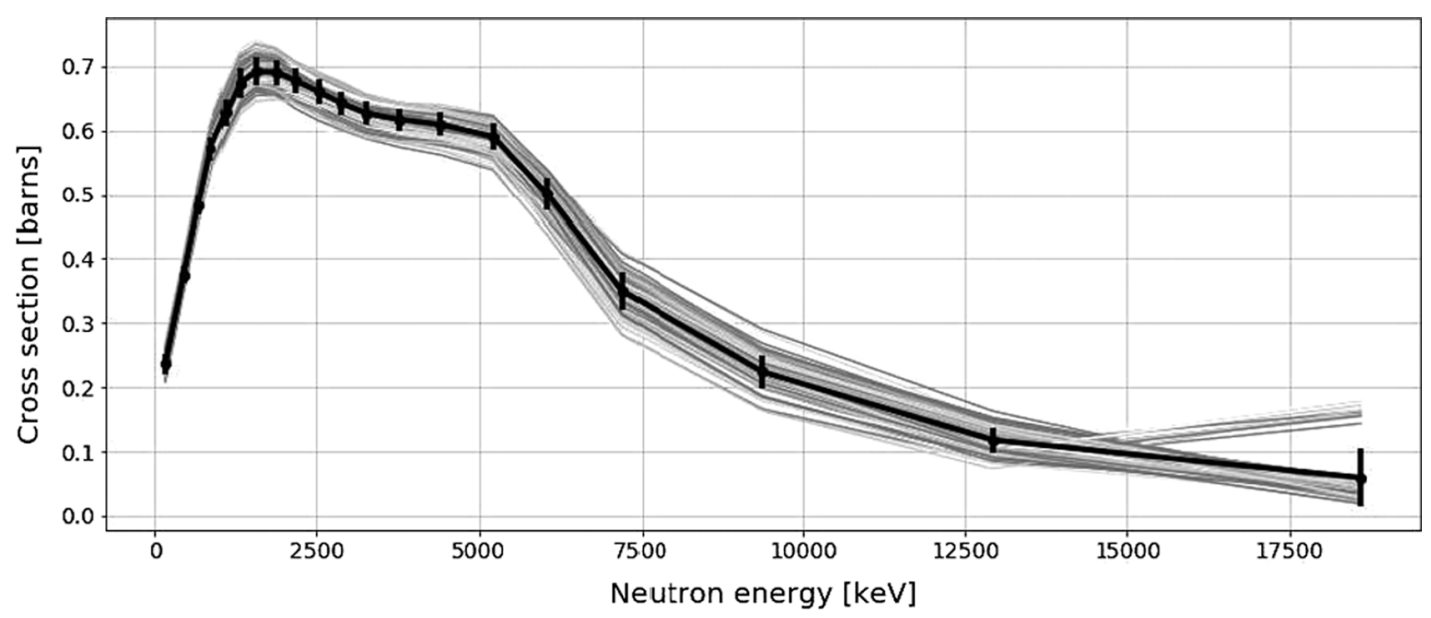

Fig. 4. $(\mathrm{n}, \mathrm{xn} \gamma)$ cross sections of the $111 \mathrm{keV} \gamma$-transition in ${ }^{184} \mathrm{~W}$ obtained with the Monte Carlo procedure. The thick black line is the final cross section associated with its standard deviation.

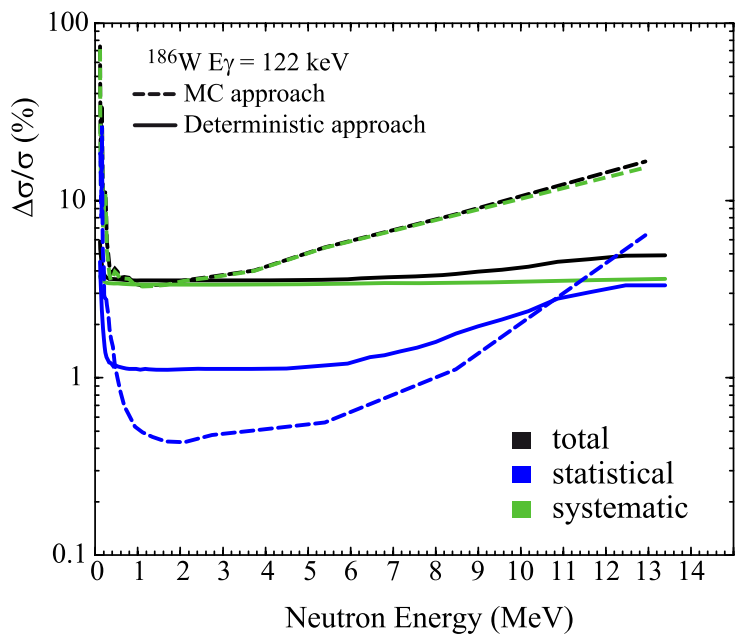

Fig. 5. Comparison of the relative uncertainties - total, statistical and systematic - of $(\mathrm{n}, \mathrm{xn} \gamma)$ cross sections obtained using the deterministic method (line) and the Monte Carlo method (dashed line) for $122 \mathrm{keV} \gamma$-transition in ${ }^{186} \mathrm{~W}$.

\section{From $(n, x n \gamma)$ to $(n, x n)$ cross sections}

\subsection{Nuclear structure sensitivity studies}

As mentioned in Section 2, well-known nuclear structure data are key ingredient for the $(\mathrm{n}, \mathrm{xn} \gamma)$ technique since the level scheme of the nucleus and other related nuclear parameters are necessary to deduce the $(\mathrm{n}, \mathrm{xn})$ cross section for any chosen way (directly from $(\mathrm{n}, \mathrm{xn} \gamma)$ cross sections or using nuclear reaction codes, see Fig. 1). When the $(\mathrm{n}, \mathrm{xn})$ cross section $\sigma_{\mathrm{nxn}}$ is deduced from the measured $(\mathrm{n}, \mathrm{xn} \gamma)$, the $(\mathrm{n}, \mathrm{xn})$ cross section is the sum of the individual level $L_{i}$ cross section $\sigma_{\mathrm{nxn}, L_{i}(E)}$ as described in the following formula:

$$
\sigma_{n x n}(E)=\sum_{i=1}^{E_{x}\left(L_{i}\right) \leq E} \sigma_{\mathrm{nxn}, L_{i}(E)}
$$

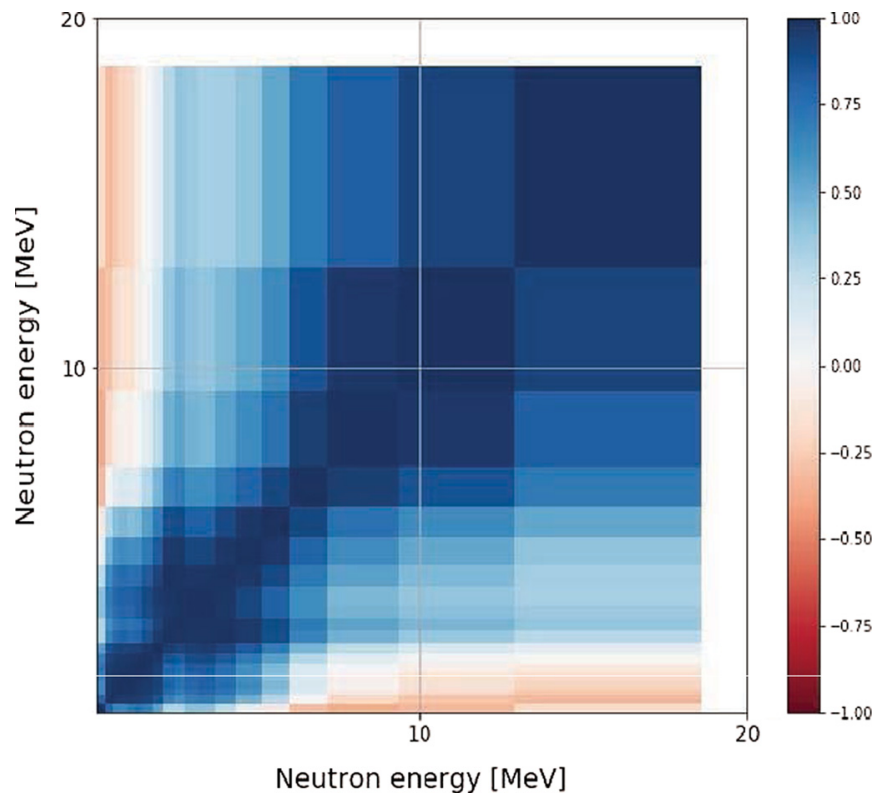

Fig. 6. Correlation matrix obtained for the $(\mathrm{n}, \mathrm{xn} \gamma)$ cross section of the $111 \mathrm{keV} \gamma$-transition in ${ }^{184} \mathrm{~W}$.

where $E_{x}$ is the excitation energy of the level $L_{i}$ and $E$ is the incident neutron energy in the center of mass system. As the level production cross section can also be expressed as a function of measured $(\mathrm{n}, \mathrm{xn} \gamma)$ cross section $\sigma_{\mathrm{nxn}, \gamma}(E$, $\left.L_{i} \rightarrow L_{j}\right), \sigma_{\mathrm{nxn}}$ can be written as:

$$
\sigma_{\mathrm{nxn}}(E)=\sum_{i=1}^{E_{x}\left(L_{i}\right) \leq E_{i n}} \sigma_{\mathrm{nxn}, \gamma}\left(E, L_{i} \rightarrow L_{j}\right) \frac{p\left(L_{i} \rightarrow g . s .\right)}{p_{\gamma}\left(L_{i} \rightarrow L_{j}\right)}
$$

where $p$ and $p_{\gamma}$ are respectively the total $(\gamma$ and internal conversion) and $\gamma$-ray emission probabilities for the transition $L_{i}$ to $L_{j}$. g.s. is the ground state. Thus this method can be applied if at least one $\gamma$-ray per excited level is observed but with the condition that level scheme and $\gamma$-transition probabilities are well-known. In the case of 

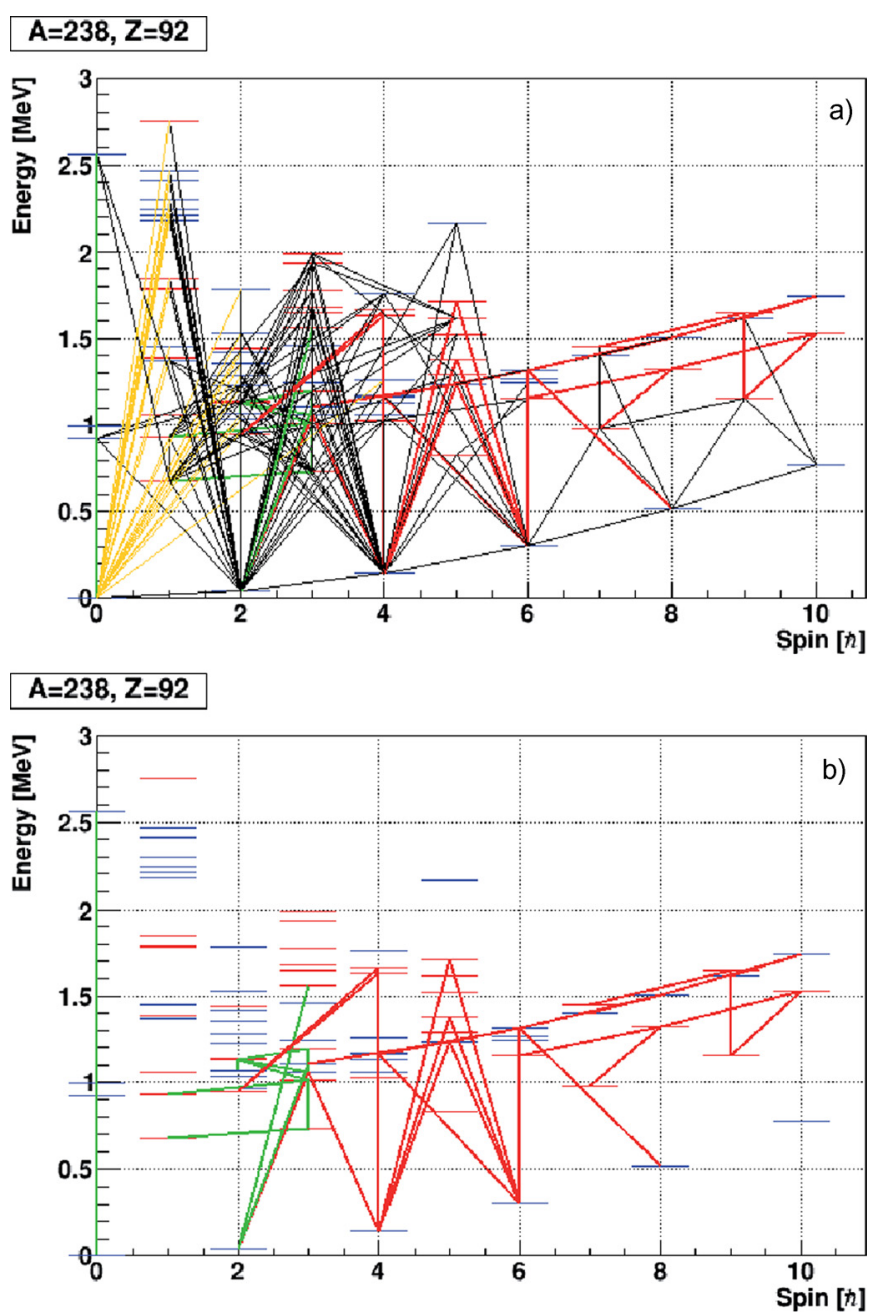

Fig. 7. Portion of the ${ }^{238} \mathrm{U}$ spin-excitation energy plan, see text for details.

${ }^{238} \mathrm{U}$ the situation is not so good, as it has been addressed in reference [11]. Indeed, in the nuclear reaction code TALYS 1.8 [12], the structure file of ${ }^{238} \mathrm{U}$ contains 152 levels among which 43 have evenly split branching ratios distributed over mainly 2 or $3 \gamma$-transitions. This reflects the present lack of knowledge on branching ratios for these levels. Even if these levels are located at high excitation energy, some of them can be populated across neutron inelastic processes and thus could impact the decay paths. This is illustrated in Figure 7a, which represents a portion of the ${ }^{238} \mathrm{U}$ spinexcitation energy plan. The levels are marked with blue (red) lines for positive (negative) parities. Known transitions are represented with black lines and the known transitions to the GS with orange lines. The green lines correspond to transitions with branching ratio equal to zero and the red ones are those for which the branching ratios are evenly splitted.

If we remove the known transitions (Fig. 7b), we see clearly that the impact on low lying levels could be important as evenly split $\gamma$ transitions redistribute the decay flux over the first and second excited states. It is thus of importance to quantify the effect of this lack of information in the nuclear structure of ${ }^{238} \mathrm{U}$. For this purpose, a first sensitivity test has been performed, with Monte Carlo technique, by testing the decay path as a function of different branching ratios sets. The first set of branching ratios was the one used in TALYS (with even split), the second was a Weisskopf estimate for even split $\gamma$-transitions, the third was a Weisskopf estimation probability for all $\gamma$-transitions probabilities and the last, an even split estimation for all $\gamma$-transitions probabilities. It sould be noted that the aim of this test is only to show the effect of different branching ratios set choices and not to obtain a realistic description. The result is that the decay path avoiding the GS band is not strongly affected by changing the branching ratio description but the paths which are connected to GS band show a dependence, estimated to about $10 \%$, on the branching ratio description. From these results, we have recently started some new sensitivity studies to better quantify the effect of the level scheme knowledge. One aim of this study is to identify which $\gamma$-transitions are the most selective for tuning the free nuclear model parameters. A first attempt has been performed with the TALYS 1.8 code for which Monte Carlo simulations have been run. Transition branching ratios have been varied independently and randomly $10 \%$ around their reference values and TALYS was used with the modified structure and default keywords. Cross sections for each transition and each run are collected and processed with the NumPy analysis package to produce the correlation matrix shown in Figure 8. Different runs have been also calculated with varied incident neutron energy to inspect the effect of excitation energy. Figure 8 corresponds to a run with an incident neutron energy of $1.3 \mathrm{MeV}$ which is more or less the excitation energy where the continuum is starting in TALYS. Moreover, in our kind of experiments, the maximum energy level from which we see $\gamma$-transitions is around $1.2 \mathrm{MeV}$ and the maximum spin is $10 \hbar$. Thus, all the $\gamma$-transitions shown in Figure 8 are involved in the (n, $\mathrm{n}^{\prime} \gamma$ ) reaction.

This correlation matrix shows, as expected, strong correlations between $\gamma$-production cross sections coming from the same excited level. Some regions are less correlated which suggests that these transitions could be used to test model predictions as the structure dependency is weak. This work on nuclear structure requires more investigations and tests to make full use of present feedback.

\subsection{Accurate $(n, x n)$ cross section deduced from modeling?}

As mentioned in the introduction, nuclear reaction codes can be used to infer the $(\mathrm{n}, \mathrm{xn})$ cross section from the measured $(\mathrm{n}, \mathrm{xn} \gamma)$ ones. Free parameters of the theoretical models are constrained by $(\mathrm{n}, \mathrm{xn} \gamma)$ cross sections. For instance, precise $(\mathrm{n}, \mathrm{xn} \gamma)$ measurements allow to probe various aspects of theoretical models and to pinpoint which particular reaction mechanism and/or nuclear structure properties (for instance branching ratio) could be described better. When phenomenological approaches are used, this could also help to perform a fine tuning of some of the models' free parameters. Models improved within this procedure are then used to predict more reliable $(\mathrm{n}, \mathrm{xn})$ 


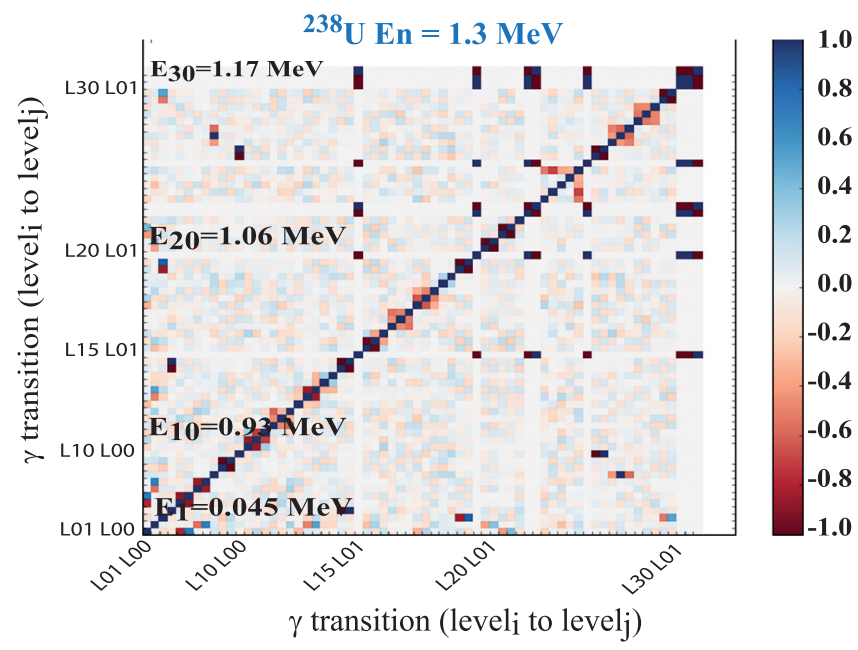

Fig. 8. Correlation matrix of $\gamma$-transition cross sections in ${ }^{238} \mathrm{U}$ $\left(\mathrm{n}, \mathrm{n}^{\prime}\right)$ reaction $(E=1.3 \mathrm{MeV})$ obtained with TALYS-1.8 where nuclear structure of ${ }^{238} \mathrm{U}$ has been varied by Monte Carlo procedure. The $\gamma$-transitions are refered to TALYS level numbering but the corresponding excitation energy is specified along the Y scale. cross sections. A good example is found in the study of the transitions within the GS band of ${ }^{238} \mathrm{U}$. Calculated cross sections performed with the TALYS code, for which the pre-equilibrium emission process is described using the phenomelogical exciton model or a microscopic reaction model built from QRPA nuclear structure information, are compared to measurements in Figure 9. Details on these calculations can be found in references [13,14]. The microscopic model better reproduces the magnitude of the transition from high spin states. This is related to the spin population of the compound nucleus which is better described within the microscopic approach. Thus, experimental data for these particular transitions constitute a magnifying glass through which specific reaction mechanisms can be seen, here the spin population after the fast and semi-rapid emission of a neutron. They thus help to challenge and improve reaction modeling. Figure 10 displays the comparison of data for interband transitions to TALYS calculations performed with various model inputs for pre-equilibrium emission (exciton/QRPA) and for E1M1 $\gamma$-strength functions (TALYS prescriptions [12]/ QRPA). The observed shape of the cross section is well accounted by the calculations, but discrepancies in

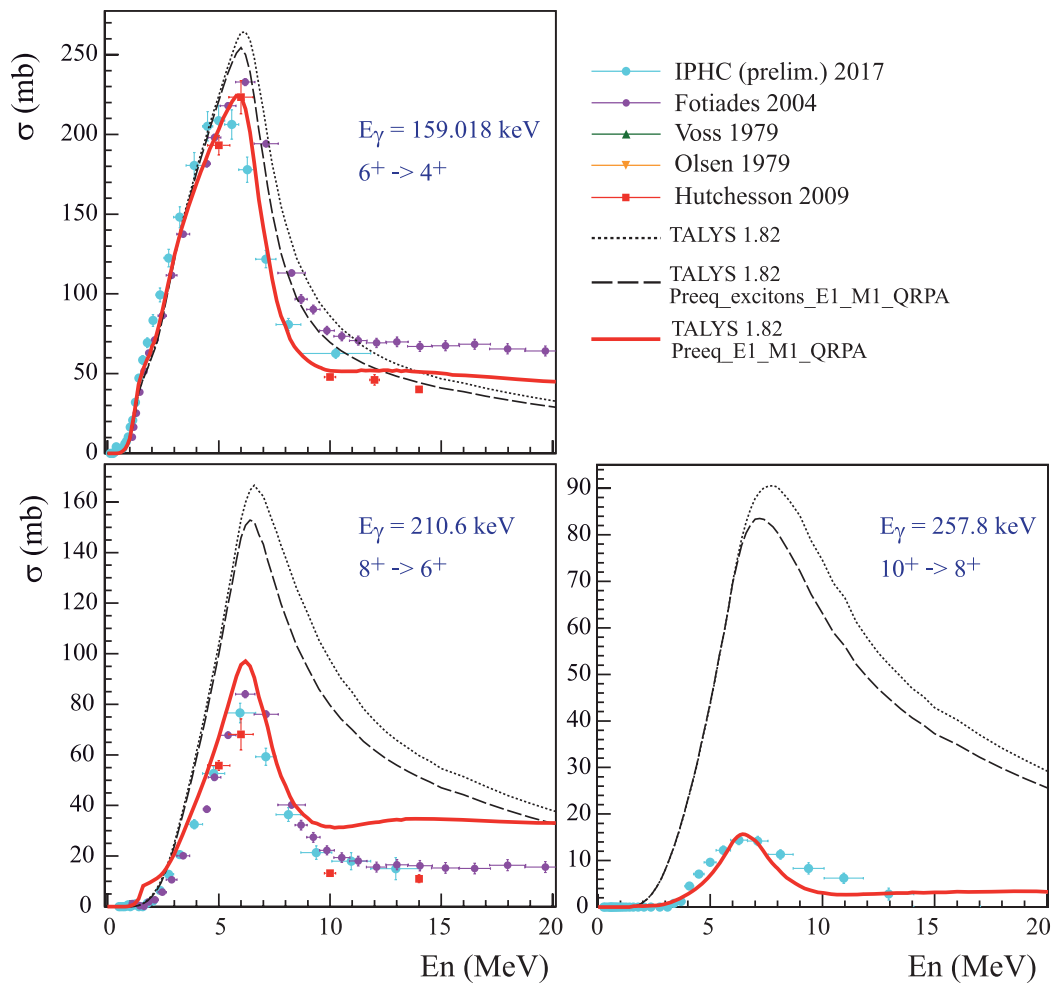

Fig. 9. Comparison between measured ${ }^{238} \mathrm{U}\left(\mathrm{n}, \mathrm{n}^{\prime} \gamma\right)$ cross sections for transitions within the ground state band and TALYS calculations with different models inputs. Our experimental data are compared to those from Hutcheson et al. [15], Fotiades et al. [16], Olsen et al. [17], Voss et al. [18]. The TALYS calculations are made either with exciton model for pre-equilibrium and E1-M1 gamma strength function from RIPL - dashed black line, or with exciton model for pre-equilibrium and E1-M1 gamma strength function from QRPA calculations - long dashed black line or with pre-equilibrium and E1-M1 gamma strength function from QRPA calculations red line. 

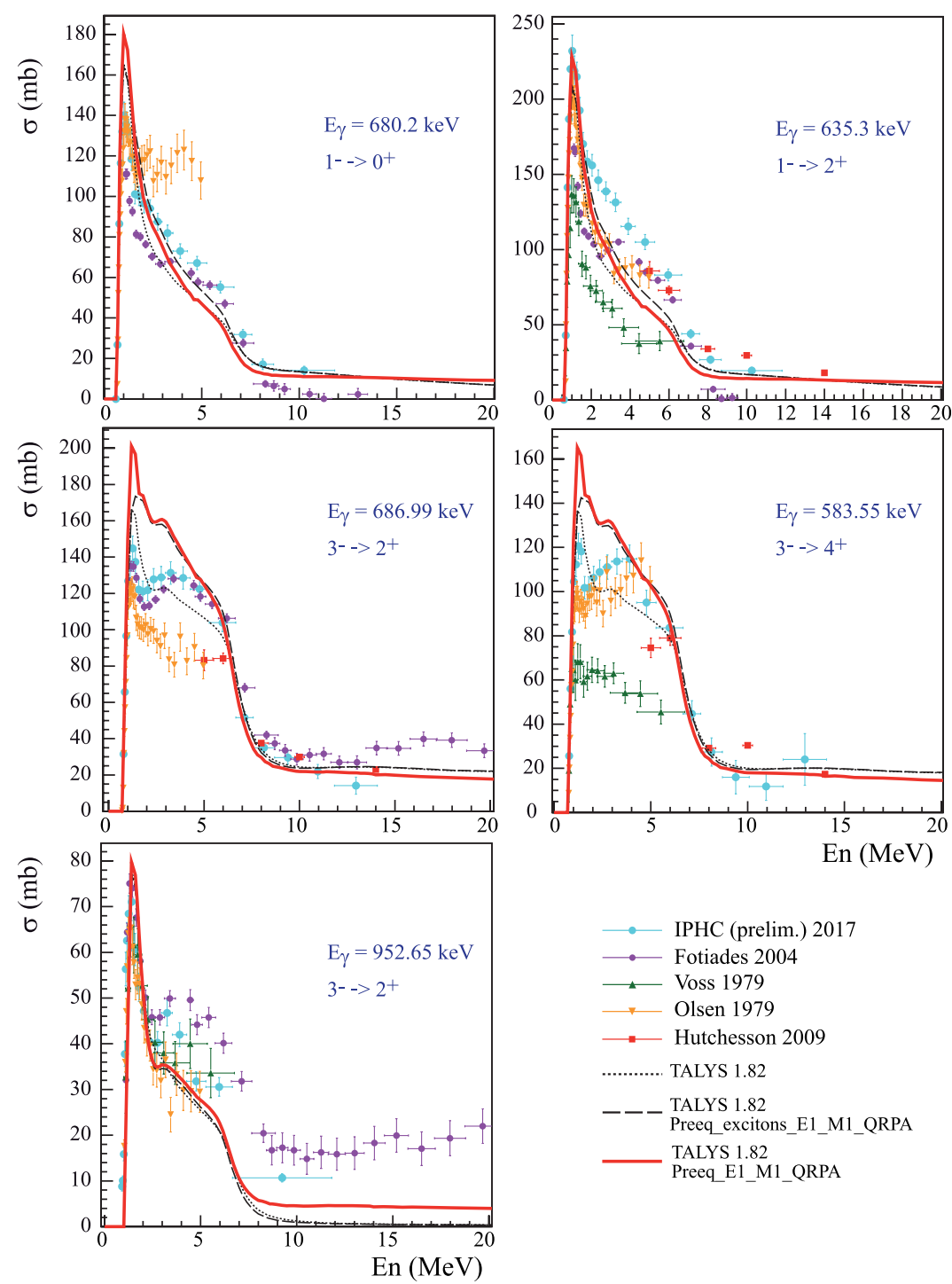

Fig. 10. Same as for Figure 8 but in the case of interband $\gamma$-transitions.

magnitude can reach 50\%. Also, for specific transitions, experimental data sets do not match. The various calculations presented here with a specific choice of models and parameters display a variation of the cross section within $10 \%$ of a mean value. However, various aspects of the modeling, such as precise knowledge of branching ratios as discussed in Section 4.1, could be modified which would result in increasing this variation. Following those considerations, the question that flows is what level of confidence could be placed in the total $(\mathrm{n}, \mathrm{xn})$ cross section calculated by tuned models and how could we quantify it? We see arising the need to produce uncertainties on theoretical predictions based on $(\mathrm{n}, \mathrm{xn} \gamma)$ cross section adjustments. These questions should be considered by theoreticians in near future to improve the $(\mathrm{n}, \mathrm{xn} \gamma)$ technique and to take advantage of the experimental effort to accurately measure the exclusive cross sections.

Another use of theoretical models is also possible when, for example, the decay of the first excited level is not observable for physical or experimental reasons (high internal conversion coefficient, low $\gamma$-energy, substantial background or contamination...). In this case, once the models are tuned on the set of $(\mathrm{n}, \mathrm{xn} \gamma)$ cross sections, the missing one is given by the nuclear reaction code and input to equation (3). This was done in reference [19] for the measurement of ${ }^{57} \mathrm{Fe}\left(\mathrm{n}, \mathrm{n}^{\prime}\right)$ cross section using the reaction code EMPIRE [20] to infer a cross section value for the $14.4 \mathrm{keV} \gamma$-transition (from the first excited level) which was not observable with the GAINS setup. An uncertainty of $10 \%$, based on the amount of overlap between all $(\mathrm{n}, \mathrm{xn} \gamma)$ cross sections calculated by EMPIRE and experimental results, was assigned to the inferred cross section. However, as discussed above and in Section 4.2 , this $10 \%$ value must be considered as partial uncertainty as they do not represent the uncertainty related to both all model ingredients and those inherent to the use of a model which deeply simplifies a dynamical many-body problem. Thus an experimental effort to measure such a cross section is deeply needed and a measurement with an uncertainty of $10 \%$ would deeply challenge the model and help improving them. 


\section{Conclusion}

The $(\mathrm{n}, \mathrm{xn} \gamma)$ technique is an indirect experimental method to measure $(\mathrm{n}, \mathrm{xn})$ reaction cross section. For several years, our collaboration has worked to manage and minimize all the uncertainties coming from the experimental procedure. The systematic uncertainty has then been reduced to $4 \%$. For the most favorable conditions, $(\mathrm{n}, \mathrm{xn} \gamma)$ cross sections are measured within $4 \%$ uncertainty but can reach up to $20 \%$ or more when statistics is unfavorable (at high neutron energy for instance). A work on the production of covariance and correlation matrices has been undertaken recently to provide very well documented experimental $(\mathrm{n}, \mathrm{xn} \gamma)$ cross sections. Current issue concerns the study of external ingredients contribution which allow the determination of $(\mathrm{n}, \mathrm{xn})$ cross section from measured $(\mathrm{n}, \mathrm{xn} \gamma)$ data. The first ingredient remains the nuclear structure information for which we have developed some Monte Carlo tools to identify its impact in the calculated (n,xn) cross section. Secondly a discussion is open with theoreticians on how to produce uncertainty information for modeled cross sections when the free parameters of models have been adjusted on $(\mathrm{n}, \mathrm{xn} \gamma)$ cross sections. Above actions are our next challenges if we aim to take advantage of the high potential of $(\mathrm{n}, \mathrm{xn} \gamma)$ technique and to provide new consolidated data sets on neutron inelastic scattering.

The authors thank the team of the GELINA facility for the preparation of the neutron beam and for their strong support day after day. This work was partially supported by NEEDS, PACEN/GEDEPEON, French ANR and by the European Commission within the Sixth Framework Programme through I3-EFNUDAT (EURATOM contract no. 036434) and NUDAME (Contract no. FP6-516487), and within the Seventh Framework Programme through EUFRAT (EURATOM contract no. FP7211499), through ANDES (EURATOM contract no. FP7249671) and through CHANDA (EURATOM contract no. FP7-605203). This work was also partially supported by a grant of the Ministery of Research and Innovation of Romania, CNCSUEFISCDI, project number PN-III-P4-ID-PCE-2016-0025 within PNCDI III.

\section{References}

1. M. Salvatores, R. Jacqmin, Nucl. Sci. -NEA/WPEC-26, Volume 26, NEA No. 6410, 2008

2. A. Santamarina, D. Bernard, P. Leconte, J.-F. Vidal, Nucl. Data Sheets 118, 118 (2014)

3. P. Romojaro, F. Alvarez-Velarde, I. Kodeli, A. Stankovskiy, C.J. Diez, O. Cabellos, N. Garcia-Herranz, J. Heyse, P. Schillebeeckx, G. Van den Eynde, G. Zerovnik, Ann. Nucl. Energy 101, 330 (2017)

4. OECD/NEA, Nuclear Data High Priority Request List, http://www.nea.fr/dbdata/hprl/
5. M. Kerveno, A. Bacquias, C. Borcea, Ph. Dessagne, G. Henning, L.C. Mihailescu, A. Negret, M. Nyman, A. Olacel, A.J.M. Plompen, C. Rouki, G. Rudolf, J.C. Thiry, Eur. Phys. J. A 51, 167, (2015)

6. M. Kerveno, J.C. Thiry, A. Bacquias, C. Borcea, P. Dessagne, J.C. Drohé, S. Goriely, S. Hilaire, E. Jericha, H. Karam, A. Negret, A. Pavlik, A.J.M. Plompen, P. Romain, C. Rouki, G. Rudolf, M. Stanoiu, Phys. Rev. C 87, 24609 (2013)

7. L.C. Mihailescu, L. Olah, C. Borcea, A.J.M. Plompen, Nucl. Instrum. Methods Phys. Res. A 531, 375 (2004)

8. D.B. Pelowitz, ed., MCNPX User's Manual, Version 2. 6. 0 , Los Alamos National Laboratory report LA-CP- 07-1473, April 2008

9. A. Negret, C. Borcea, Ph. Dessagne, M. Kerveno, A. Olacel, A.J.M. Plompen, M. Stanoiu, Phys. Rev. C 90, 034602 (2014)

10. NumPy, Base N-dimensional array package, https://docs. scipy.org/doc/numpy-1.10.1/reference/generated/numpy. cov.html

11. G. Henning, Workshop on experimental and theoretical problematic around actinides for future reactors. Espace de Structure Nucléaire Théorique (CEA/DSM-DAM), Saclay, 17-19 March 2014, http://esnt.cea.fr/Phocea/Page/index. php?id $=37$

12. A.J. Koning, S. Hilaire, M.C. Duijvestijn, TALYS-1.0, in Proceedings of the International Conference on Nuclear Data for Science and Technology, April 22-27, 2007, Nice, France, edited by O. Bersillon, F. Gunsing, E. Bauge, R. Jacqmin, S. Leray (EDP Sciences, 2008), pp. 211-214

13. M. Dupuis, E. Bauge, S. Hilaire, F. Lechaftois, S. Péru, N. Pillet, C. Robin, Eur. Phys. J. A 51, 168 (2015)

14. M. Dupuis, S. Hilaire, S. Péru, E. Bauge, M. Kerveno, P. Dessagne, G. Henning, EPJ Web Conf. 146, 12002 (2017)

15. A. Hutcheson, C. Angell, J.A. Becker, A.S. Crowell, D. Dashdorj, B. Fallin, N. Fotiades, C.R. Howell, H.J. Karwowski, T. Kawano, J.H. Kelley, E. Kwan, R.A. Macri, R.O. Nelson, R.S. Pedroni, A.P. Tonchev, W. Tornow, Phys. Rev. C 80, 014603 (2009)

16. N. Fotiades, G.D. Johns, R.O. Nelson, M.B. Chadwick, M. Devlin, M.S. Wilburn, P.G. Young, J.A. Becker, D.E. Archer, L.A. Bernstein, P.E. Garrett, C.A. McGrath, D.P. McNabb, W. Younes, Phys. Rev. C 69, 024601 (2004)

17. D.K. Olsen, G.L. Morgan, J.W. McConnell, in Proceedings of the International Conference on Nuclear Cross Sections for Technology: Knoxville, TN, 22 Oct. 1979, edited by J.L. Fowler, C.H. Johnson, C.D. Bowman (National Bureau of Standards, Washington, 1979), p. 677

18. F. Voss, S. Cierjacks, D. Erbe, G. Schmatz, Kernforschungszentrum Karlsruhe Report No. 2379, (1976) (unpublished)

19. A. Negret, M. Sin, C. Borcea, R. Capote, Ph. Dessagne, M. Kerveno, N. Nankov, A. Olacel, A.J.M. Plompen, C. Rouki Phys. Rev. C 96, 024620 (2017)

20. M. Herman, R. Capote, M. Sin, A. Trkov, B.V. Carlson, P. Oblozinsky, C.M. Mattoon, H. Wienkey, S. Hoblit Young-Sik Cho, G.P.A. Nobre, V. Plujko, V. Zerkin, empire-3.2 (Malta) Users Manual, report INDC(NDS)-0603 of the International Atomic Energy Agency, Vienna, 2013

Cite this article as: Maëlle Kerveno, Greg Henning, Catalin Borcea, Philippe Dessagne, Marc Dupuis, Stéphane Hilaire, Alexandru Negret, Markus Nyman, Adina Olacel, Eliot Party, Arjan Plompen, How to produce accurate inelastic cross sections from an indirect measurement method? EPJ Nuclear Sci. Technol. 4, 23 (2018) 